\title{
Los estilos de enseñanza: construyendo puentes para transitar las diferencias individuales del alumnado.
}

\section{Teaching styles: building bridges to approach individual differences of students}

\author{
Margarita GONZÁLEZ-PEITEADO y Margarita PINO-JUSTE \\ UNED y Universidad de Vigo
}

Recibido: Diciembre 2014

Evaluado: Febrero 2015

Aceptado: Marzo 2015

\section{Resumen}

Para progresar en el camino hacia una efectiva atención a la diversidad, es inevitable desarrollar un estilo de enseñanza que tenga en cuenta las diferencias individuales que presenta el alumnado, muy vinculadas a intereses, necesidades, aptitudes, potencialidades y estilos de aprendizaje particulares del educando. Rasgos, todos ellos, que los hace únicos y diferenciados. Así, desde el estudio de la variabilidad intersujeto y partiendo de un marco teórico multidimensional, se describen y analizan las diferencias interindividuales e intergrupales de 555 estudiantes de la Facultades de Ciencias de la Educación de Galicia pertenecientes a las Universidades de Coruña, Santiago de Compostela y Vigo, bajo el prisma de los siguientes estilos de enseñanza: reflexivo, cooperador, individualizador, innovador, indagador y académico. Los resultados obtenidos revelan la existencia de diferencias significativas en las preferencias sobre estilos de enseñanza en función de las variables propuestas: Universidad, especialidad, sexo y edad.

Palabras clave: estilos de enseñanza, diferencias individuales, educación, formación inicial, profesorado.

\begin{abstract}
We are aware that in education, if we want to progress towards an effective attention to diversity, it is unavoidable to develop teaching methods that take into account pupils' individual differences, linked to their interests, necessities, aptitudes, potentialities and particular learning styles. All these features make them unique and differentiated. From the study of inter-personal variability and based on a multidimensional theoretical framework, we describe and analyse interindividual and inter-group differences of 555 students from the Faculties of Education Sciences of Galicia, belonging to the Universities of La Coruña, Santiago de Compostela and Vigo, under the focus of the following different teaching styles: reflexive, cooperative, individualizer, innovative, researcher and academic. The results obtained reveal the existence of significant differences in the preferences on teaching styles according to the four variables posed: University, specialty, sex and age.
\end{abstract}

Keywords: teaching styles, individual differences, education, initial training, teacher. 
Los docentes tienden a manifestar en el ejercicio de su profesión verdaderas construcciones simbólicas que conforman sistemas de valores, ideas, técnicas y métodos que articulados se convierten en su particular estilo de enseñar (GonzálezPeiteado, 2012). Es así, como los estilos de enseñanza presuponen la existencia de diferencias individuales en los profesionales de la educación y dotan de unidad y coherencia a la actuación docente. Se hace referencia a una cierta regularidad en el comportamiento a lo largo de diversas situaciones y a una relativa constancia transituacional influenciada por las características personales y psicológicas del docente dentro de la dinámica que se establece en el aula.

No se puede abordar el análisis de los estilos de enseñanza sin detenerse en considerar la singularidad y variabilidad existente entre individuos debido a que según sea el estilo docente, éste se relacionará con los distintos elementos del acto didáctico de una forma que marcará las propias relaciones con los mismos influyendo de manera significativa en el aprendizaje de los alumnos y la calidad del proceso.

Dicha diferencia gira en torno a constructos cognitivos, biológicos, culturales, dimensiones situacionales, experiencia académica y teorías de la enseñanza que sustentan la práctica educativa. Es desde la aceptación de esta diversidad como puede comprenderse que cada persona presente una determinada forma de enseñar y de aprender, manifestando una variedad de conductas y roles a lo largo del proceso educativo, contribuyendo a la conformación de un estilo determinado. Por tanto cuando se habla de estilos de enseñanza se alude al modo particular, relativamente estable que posee cada profesor al abordar el proceso de enseñanza-aprendizaje (GonzálezPeiteado y Pino-Juste, 2014). Es la forma peculiar de atender al alumnado, de conducirlo hacia el aprendizaje, de percibir sus necesidades, intereses y aptitudes, de interactuar y pensar acerca del acto educativo haciéndose patente a través de las concepciones, sentimientos y supuestos que el docente sostiene preferentemente en el ejercicio de su profesión. Ello se ve reflejado en la organización y gestión de la clase, en los métodos y los recursos que utiliza, en la expresión oral y corporal (GonzálezPeiteado, 2013).

A este respecto, se señala la existencia histórica de dos grandes estilos: El estilo de enseñanza académico y el estilo de enseñanza activo que según el momento y el contexto fueron adoptando diferentes denominaciones. Así podemos mencionar las experiencias realizadas por Flanders, 1977 y más recientemente las llevadas a cabo por Gargallo, 2008-2010; Hood, Poulson, Mason, Walker y Dixon, 2009; Michel, James y Varela, 2009; Weltman y Whiteside, 2010 y González-Peiteado (2012).

Otros autores como Delgado (1996), amplían el número de factores hasta 6 aumentando el abanico de comportamientos. 


\begin{tabular}{ll}
\hline \multicolumn{1}{c}{ AUTOR } & \multicolumn{1}{c}{ CLASIFICACIÓN } \\
\hline Flanders (1977) & $\begin{array}{l}\text { Estilo directo (tradicional)/estilo indirecto (espontaneidad y } \\
\text { diálogo) }\end{array}$ \\
\hline Delgado Noguera (1996) & $\begin{array}{l}\text { Estilo tradicional/individualizador/participativo/ } \\
\text { socializador/cognoscitivo/creador }\end{array}$ \\
\hline Gargallo López (2008) & Estilo centrado en la enseñanza/estilo centrado en la tarea \\
\hline Coldrey y Hively (2009) & Authoritarian style/authoritative style \\
\hline Michel, James, Varela (2009) & Estilo activo/estilo pasivo \\
\hline Weltman y Whiteside (2010) & Estilo transaccional/Estilo transformacional \\
\hline
\end{tabular}

Cuadro 1. Clasificaciones de los estilos de enseñanza en función del autor.

Fuente: Elaboración propia.

Al pensar en abordar la cuestión de las representaciones del rol docente, se habla de un espacio pedagógico en el que cada educador construye procesos de análisis y reflexión constante sobre sí mismo y sobre el contexto de enseñanza con el propósito de diagnosticar, prevenir y en caso necesario intervenir reorientando y adaptando el proceso de enseñanza-aprendizaje. Por tanto, es imprescindible adoptar actitudes de responsabilidad, compromiso personal y competencias en relación al saber, saber hacer, saber ser y saber desaprender. A lo que se añade la inquietud permanente por optimizar el desarrollo de la acción educativa dando respuesta a un marco educativo que cuestiona los modelos tradicionales de enseñanza y persigue la excelencia.

Bajo este planteamiento, los roles tradicionalmente asumidos por los docentes, poco permeables a considerar las diferencias y la diversidad en las aulas, tendentes a homogeneizar la realidad y a crear estereotipos, resultan obviamente inadecuados. Sin embargo, en ocasiones la escuela no contribuye eficazmente al desarrollo de la creatividad, la individualización de la enseñanza, la capacidad reflexiva y de indagación, a aprender a aprender y a aprender a pensar. Es preciso ser consciente de que "está muy arraigado el modelo por antonomasia de la docencia tradicional centrada en la enseñanza donde el profesor es el protagonista y los alumnos receptores pasivos de información. Información que por otro lado, ha sido seleccionada, preparada y elaborada, por y con los criterios del profesor. El alumno, por tanto no hace más que aceptarla, memorizarla y, en el mejor de los casos, intentar comprenderla. Desde este punto de vista, el alumno recibe la visión parcial y subjetiva que el profesor le propone" (Ballesta, Izquierdo, Romero, 2011:356).

Por otra parte, las estrategias de enseñanza están en correspondencia con el estilo de aprendizaje de cada docente, por lo que es importante que se adquiera conciencia de ello y se pueda medir su impacto en el modo de aprender de los estudiantes (Aguilera y Ortiz, 2010; González-Peiteado y Rodríguez-López, 2014).

Curiosamente, a medida que avanza el proceso educativo, la creatividad disminuye en el alumnado y se vuelven menos activos (Martínez Geijo, 2007) debido a la baja preferencia del profesorado por dicho estilo (Raposo, Barcia, Fernández-Carballido, Montejo, Negro, y Gallego, 2004). En otras investigaciones se sostiene que los docentes tienden a desarrollar el estilo reflexivo en detrimento de otros estilos como el creativo (López Aguado, 2011). 


\section{¿Problemas de enseñanza o problemas de aprendizaje?}

Considerando que enseñar es una actividad de facilitación del aprendizaje, cuyo sostenimiento se asienta en el compromiso del docente con la finalidad educativa y con el alumno; el profesor debe crear las condiciones adecuadas para hacer realidad los postulados de un estilo de enseñanza activo, atendiendo tanto a la singularidad como a la diversidad del alumnado. Sin embargo, ¿Por qué este cambio cualitativo no siempre se refleja en las aulas? A menudo observamos como un estilo de enseñanza tradicionalreproductivo, centrado en el profesor y las actuaciones homogeneizadoras coexisten con argumentos políticamente correctos de atención a la diversidad.

Abundando en la cuestión cabe preguntarse entonces, ¿Por qué habitualmente se señalan problemas de aprendizaje y difícilmente se habla de problemas de enseñanza? Si el profesor enseña ¿Por qué hay alumnos que no aprenden? ¿Puede el estilo docente contribuir a generar un problema? Consideramos que estos interrogantes, en función de las premisas antes expuestas, demuestran la importancia de conocer cuáles son los estilos de enseñanza de los futuros profesionales de la educación ya que la praxis docente se hace patente a la hora de observar con detenimiento la realidad escolar: la organización del aula, el control establecido, el nivel de exigencia, el grado de comunicación y empatía, el uso de formas eficaces de motivar y captar la atención del alumnado. A este respecto, el aprendizaje de los alumnos depende de lo que los profesores saben y de lo que pueden hacer (Darling-Hammond, 2000).

Existen evidencias que demuestran que el aprendizaje puede mejorar ajustando el estilo de enseñanza al estilo de aprendizaje del alumno (Alonso, 1999; Gallego Gil y Nevot Luna, 2008, Martínez Geijo, 2008). Por tanto, teniendo en cuenta que la falta de congruencia entre estilos de enseñanza y estilos de aprendizaje pueden predisponer al fracaso escolar (Cabrera et al, 2006), las actuaciones docentes deben contemplar las diferencias en la forma de abordar el aprendizaje por parte de los alumnos, con el objetivo de poder ajustar su plan de actuación y optimizar así el aprendizaje de todos los alumnos (Alonso, 2008; López Aguado, 2011, Tejada, 2011). Para lograr este propósito, los profesores deben conocer el perfil del alumno y del grupo en general, como la manera de implementar metodologías y estilos eficaces, que conduzcan al logro del aprendizaje. Sin embargo, puede suceder que los docentes identifiquen las peculiaridades de cada alumno al enfrentarse al aprendizaje, pero que no dispongan de los recursos y destrezas que les permitan atender esa diversidad lo que complica su trabajo, suponiendo un esfuerzo añadido.

Un docente eficiente es aquel capaz de asumir el desafío de formar personas competentes respetando su singularidad. Para ello, se hace imprescindible reflexionar sobre aquellas estrategias que optimizan su particular estilo de enseñanza en un intento por ajustarlo al estilo de aprendizaje del discente (Gravini Cabrera, Ávila y Vargas, 2009).

Concretamente, a través del presente estudio, pretendemos aproximarnos al conocimiento diferencial de los estilos de enseñanza preferidos por los alumnos de las facultades de Ciencias de la Educación de la Comunidad de Galicia. Entendiendo por estilos de enseñanza no sólo como la forma peculiar de conducir la clase, sino también, 
como el modo preferente de interactuar profesorado y alumnado, de provocar oportunidades de aprendizaje y experiencias compatibles con el bienestar psicológico, la motivación y el gusto por el trabajo bien hecho.

La tipología desarrollada en el presente estudio está inspirada en la clasificación elaborada por Delgado (1996). Se ha estimado interesante someter a estudio un espectro amplio de estilos, debido a que en el comportamiento docente intervienen variables que contribuyen a que surjan factores intermedios.

\begin{tabular}{|c|c|}
\hline Estilos & Características \\
\hline Estilo académico & $\begin{array}{l}\text { Se sustentan en el orden, la tarea y el control exhaustivo. } \\
\text { El docente es quien tomas las decisiones. } \\
\text { Promueve la pasividad del alumnado. } \\
\text { No existe individualización sino una respuesta colectiva, con la misma tarea } \\
\text { e intensidad. } \\
\text { No existen relaciones afectivas } \\
\text { No existe individualización }\end{array}$ \\
\hline $\begin{array}{l}\text { Estilo } \\
\text { Individualizador }\end{array}$ & $\begin{array}{l}\text { Se pretende atender individualmente al alumno y proporcionarle una } \\
\text { enseñanza que tenga en cuenta sus capacidades, intereses y motivaciones. } \\
\text { La enseñanza se adapta al período evolutivo del discente y al grado de } \\
\text { madurez adquirida. } \\
\text { La organización del espacio y del tiempo debe permitir actividades } \\
\text { individuales y grupales. } \\
\text { Flexibilidad en los programas. } \\
\text { Docente recurre a la retroalimentación inmediata. }\end{array}$ \\
\hline Estilo Reflexivo & $\begin{array}{l}\text { Provoca mayor autonomía en el alumnado. } \\
\text { Intervienen los alumnos en la toma de decisiones. } \\
\text { Se favorece la implicación de niños/as en el proceso de aprendizaje: } \\
\text { Aprender a pensar } \\
\text { Se concede más libertad al alumno en procura del facilitar mayor capacidad } \\
\text { analítica, independencia de criterio y espontaneidad. }\end{array}$ \\
\hline Estilo Cooperador & $\begin{array}{l}\text { Estimula la cooperación, la solidaridad, a través de la práctica del trabajo } \\
\text { grupal. } \\
\text { Fomenta la responsabilidad y un clima de cordialidad. }\end{array}$ \\
\hline Estilo Indagador & $\begin{array}{l}\text { El docente es un guía. } \\
\text { Se fomentan actitudes de curiosidad e indagación. } \\
\text { Se favorece el aprendizaje por descubrimiento, la capacidad crítica y la toma } \\
\text { de decisiones. } \\
\text { Se transfieres a situaciones reales el proceso de resolución de problemas. }\end{array}$ \\
\hline Estilo Innovador & $\begin{array}{l}\text { Se busca conseguir un aprendizaje activo y espontáneo. } \\
\text { Respeto por el empleo de respuestas inusitadas e ideas originales }\end{array}$ \\
\hline
\end{tabular}

Cuadro 2. Características de los estilos de enseñanza.

Fuente: Elaboración propia. 
En consecuencia, los estilos de enseñanza están categorizados en función de las siguientes dimensiones: estilo académico y estilo activo. Este último integrado por los estilos reflexivo, cooperador, individualista, innovador e indagador.

Es importante que el profesor los conozca para poder favorecer el proceso de enseñanza-aprendizaje. Son muchos los trabajos que hacen énfasis en que cada persona posee un estilo característico, relativamente estable pero que necesariamente debe ser combinado con otros estilos para poder adecuarse a las singularidades del alumnado. (Delgado, 1992; Hervás, 2005).

En todo caso, el objetivo de este estudio radica en realizar una aproximación descriptiva e inferencial para conocer las tendencias de los estudiantes considerando variables como la Universidad en la que estudian, la especialidad que cursan, el sexo y la edad, con el fin de obtener parámetros estadísticos que caractericen a tales grupos. Así, con un propósito indagador, hemos establecido los siguientes hilos conductores de nuestro estudio:

- ¿Hay diferencias significativas en los estilos de enseñanza preferidos por los estudiantes en función de la universidad?

- ¿La especialidad determina un estilo de enseñanza preferido?

- ¿El sexo determina la preferencia hacia un estilo de enseñanza u otro?

- ¿La tendencia hacia un determinado estilo varía significativamente en función de la edad?

\section{Método}

En un intento de aproximación a la realidad educativa, se ha llevado a cabo un estudio descriptivo e inferencial de carácter transversal con el fin de conocer los estilos de enseñanza preferidos por los alumnos de $3^{\circ}$ curso de las Facultades de Ciencias de la Educación de Galicia, atendiendo a su dimensión simbólica, a su actitud, a las relaciones existentes y modos de comunicación en el aula. Se parte del hecho de que los estilos de enseñanza oscilan dentro de un continuum, puesto que prácticamente nunca se dan estilos puros, pero si la existencia de un estilo predominante que puede aprenderse, variar o mejorar conforme avanzan en el proceso de enseñanza.

En primera instancia se llevó a cabo un estudio descriptivo para identificar las características de la muestra y de las variables objeto de estudio. Se obtuvieron frecuencias y descriptivos (medias y desviaciones típicas). Seguidamente, se estudiaron las diferencias entre medias aplicando la t-Student como prueba paramétrica para dos muestras independientes, el análisis de varianza de un factor como prueba paramétrica para K muestras independientes y el HSD de Tukey para comparaciones múltiples.

\section{Participantes}

La población de estudio se encuentra establecida por los estudiantes de tercer curso de las Facultades de Ciencias de la Educación de la Comunidad de Galicia pertenecientes a la Universidad de Vigo, a la Universidad de Santiago y a la 
Universidad de Coruña, con un total de 1314 estudiantes que se reparten entre las especialidades de Educación Infantil, Educación Primaria, Educación Musical, Educación Física y Lenguas extranjeras. En base a que la determinación de que el tamaño teórico de la muestra se obtiene en función de tres valores: varianza poblacional, nivel de confianza y el máximo error permitido en las estimaciones se ha procedido al cálculo muestral $(\mathrm{N}=492)$. Resultado que lleva a confirmar que el tamaño de la muestra del presente estudio $(\mathrm{N}=555)$ es suficientemente amplia.

Se ha conseguido la existencia de representatividad en las tres Universidades y como era de esperar los mayores porcentajes de alumnos por especialidades se registran en Educación Infantil y Educación Primaria ya que son especialidades que se imparten en las tres Universidades.

\begin{tabular}{llllllllllllll}
\hline \multicolumn{4}{c}{ Datos académicos } & \multicolumn{4}{c}{ Datos personales } \\
\hline \multicolumn{3}{c}{ Universidad } & \multicolumn{4}{c}{ Especialidad \% } & \multicolumn{3}{c}{ Sexo \% } & \multicolumn{3}{c}{ Edad \% } \\
\hline & $\mathbf{N}$ & $\mathbf{\%}$ & EI & EP & EF & EM & LE & H & M & $\mathbf{- 2 0}$ & $\mathbf{2 1 - 2 3}$ & $+\mathbf{2 4}$ \\
\hline Uvigo & $\mathbf{3 3 2}$ & $\mathbf{5 9 . 8}$ & 29.4 & 17.1 & 5.6 & 4.9 & 2.9 & 17.2 & 82.8 & 42.5 & 41.5 & 16.0 \\
\hline USC & $\mathbf{1 5 9}$ & $\mathbf{2 8 . 6}$ & 07.4 & 09.4 & 4.1 & 2.5 & 5.2 & 22.6 & 77.4 & 26.4 & 48.4 & 25.2 \\
\hline UdC & $\mathbf{0 6 4}$ & $\mathbf{1 2 . 6}$ & 04.5 & 03.1 & 4.1 & 0.0 & 0.0 & 15.6 & 84.4 & 25.0 & 62.5 & 12.5 \\
\hline Total & $\mathbf{5 5 5}$ & $\mathbf{1 0 0}$ & 41.3 & 29.5 & 10.8 & 7.4 & 11.0 & & & & &
\end{tabular}

Tabla 1. Datos académicos y personales de la muestra.

Entre los datos de la muestra, hemos de precisar que la mayor presencia de alumnos en la muestra es de la Universidad de Vigo (59.8\%), seguido de la Universidad de Santiago (28.6\%). Con los menores porcentajes se encuentra la Universidad de Coruña (12.5\%), debido entre otras causas al menor número de especialidades que imparte.

En lo referente al sexo, existe una mayoría de mujeres (81.4\%) respecto a los hombres (18.6\%) que lejos de ser un sesgo en esta muestra, es coherente con los estándares ordinarios de presencia de hombres y mujeres en las aulas de las Facultades de Ciencias de la Educación.

La edad se distribuye de la siguiente forma: El 35.9\% de la muestra tiene menos de 20 años, el $45.9 \%$ integra la franja de 21 a 23 años, mientras que el $18.2 \%$ posee más de 24 años. Este dato esta en concordancia con los patrones tipo del estudiante de magisterio en nuestro país.

Si consideramos la especialidad, observamos que el intervalo con mayor porcentaje de estudiantes es el de 21 a 23 años en todas las especialidades, seguido del tramo de menos de 20 años y finalmente los alumnos con edades superiores a los 24 años.

\section{Instrumento}

En función de los objetivos formulados y del diseño establecido, se aplicó la escala sobre estilos de enseñanza (ESEE) de elaboración propia con el propósito de conocer las preferencias que manifiestan los estudiantes. El análisis de las propiedades 
psicométricas y estadísticas de los ítems nos proporciona una escala compuesta por 31 ítems y seis estilos de enseñanza, revelando que se trata de un instrumento psicométricamente adecuado para la valoración de los estilos docentes (GonzálezPeiteado, López-Castedo, Pino-Juste, 2013). Para cumplimentar el cuestionario, cada estudiante debía elegir entre cuatro alternativas en la que 0 significa totalmente en desacuerdo y 4 totalmente de acuerdo.

La escala en tanto que recurso cuantitativo ha permitido a) hallar en cada ítem una puntuación que nos aproxime al estilo de enseñanza del alumno, b) conocer el grado de incidencia de diversas variables sobre los estilos de enseñanza, c) emplear programas estadísticos para reducir costes y esfuerzo en el manejo de datos. Todo ello con un doble propósito: a) a corto plazo, conocer y describir las percepciones e imágenes conformadas en relación a los estilos de enseñanza, con la intención de introducir mejoras en el desarrollo práctico docente desde las facultades de Ciencias de la Educación. b) en todo momento, sensibilizar a la comunidad educativa hacia la autoevaluación y autocrítica de su labor en el aula.

\section{Procedimiento}

La estrategia básica de aplicación del instrumento consistió en administrar la escala a los centros personalmente, haciendo constar la participación anónima, voluntaria y la confidencialidad de la información.

\section{Análisis de datos}

Con la finalidad de determinar las características de la consistencia interna de los diversos ítems de un mismo factor y de la escala en su conjunto y la objetividad de la medida, se calculó la fiabilidad mediante el coeficiente alfa de Cronbach ofreciéndonos una adecuada fiabilidad $(\alpha=.886)$ para el conjunto de la escala y superior a $\alpha=.600$ en todas las dimensiones.

\begin{tabular}{|l|r|r|r|r|r|r|}
\hline Factores & Factor 1 & Factor 2 & Factor 3 & Factor 4 & Factor 5 & Factor 6 \\
\hline Etiqueta & Reflexivo & Cooperador & Académico & Individualizador & Innovador & Indagador \\
\hline $\begin{array}{l}\text { Alpha de } \\
\text { Cronbach }\end{array}$ & $\alpha .797$ & $\alpha .780$ & $\alpha .791$ & $\alpha .732$ & $\alpha .600$ & $\alpha .626$ \\
\hline
\end{tabular}

Tabla 2. Fiabilidad de los factores estudiados.

\section{Resultados}

En un primer análisis global, observamos una baja puntuación en las medias correspondientes a los ítems del estilo de enseñanza académico. Por lo que en líneas generales los resultados se posicionan a favor de actitudes vinculadas a estilos de enseñanza activos, apoyados en la combinación de cinco variables: Estilos de enseñanza reflexivo, cooperador, individualizador, innovador e indagador.

\begin{tabular}{lllllll}
\hline $\begin{array}{l}\text { Estilos de } \\
\text { enseñanza }\end{array}$ & $\begin{array}{l}\text { Estilo } \\
\text { Reflexivo }\end{array}$ & $\begin{array}{l}\text { Estilo } \\
\text { Cooperador }\end{array}$ & $\begin{array}{l}\text { Estilo } \\
\text { Académico }\end{array}$ & $\begin{array}{l}\text { Estilo } \\
\text { Individualizador }\end{array}$ & $\begin{array}{l}\text { Estilo } \\
\text { Innovador }\end{array}$ & $\begin{array}{l}\text { Estilo } \\
\text { Indagador }\end{array}$ \\
\hline Media & 3.46 & 3.58 & 1.82 & 3.36 & 3.70 & 3.35 \\
\hline Desv. típ. & .59 & .55 & .79 & .64 & .50 & .57 \\
\hline
\end{tabular}

Tabla 3. Estadísticos descriptivos. 
Seguidamente se lleva a cabo un análisis inferencial. Los estadísticos empleados en el estudio de las diferencias significativas van a variar en función del tipo de variable con que se trabaje. Utilizamos un diseño unifactorial, aplicando la prueba $\mathrm{T}$, cuando se trata de dos grupos y un análisis de varianza (Anova) de un factor cuando se trata de multigrupos. Para las comparaciones post hoc, se recurre al empleo de la prueba Tukey.

En la tabla 4 se recogen resultados que revelan que la variable universidad es una fuente significativa de variación, presentando diferencias significativas en el estilo reflexivo y el estilo académico entre la Universidad de Vigo y la Universidad de Santiago, siendo ésta la que evidencia una mayor tendencia hacia un estilo de enseñanza reflexivo y puntuaciones menos coherentes con un estilo académico.

\begin{tabular}{|c|c|c|c|c|}
\hline FACTORES & Media & $\mathbf{F}$ & Sig. & HSD de Tukey Sig.* \\
\hline \multirow[t]{3}{*}{ E. reflexivo } & U.Vigo 27.50 & 3.878 & .021 & \multirow{3}{*}{ Uvigo-USC.: .015 } \\
\hline & U.S.C. 28.28 & & & \\
\hline & U.Coruña 27.68 & & & \\
\hline \multirow[t]{3}{*}{ E. cooperador } & U.Vigo 25.13 & \multirow[t]{3}{*}{.582} & \multirow[t]{3}{*}{.559} & \\
\hline & U.S.C. 24.89 & & & \\
\hline & U.Coruña 25.16 & & & \\
\hline \multirow[t]{3}{*}{ E. académico } & U.Vigo 13.10 & \multirow[t]{3}{*}{3.699} & \multirow[t]{3}{*}{.025} & \multirow[t]{3}{*}{ Uvigo-USC.: .033 } \\
\hline & U.S.C. 12.28 & & & \\
\hline & U.Coruña 12.37 & & & \\
\hline \multirow[t]{3}{*}{ E. individualizado } & U.Vigo 16.74 & \multirow[t]{3}{*}{.572} & \multirow[t]{3}{*}{.565} & \\
\hline & U.S.C. 16.95 & & & \\
\hline & U.Coruña 16.91 & & & \\
\hline \multirow[t]{3}{*}{ E. innovador } & U.Vigo 7.37 & \multirow[t]{3}{*}{.889} & \multirow[t]{3}{*}{.412} & \\
\hline & U.S.C. 7.46 & & & \\
\hline & U.Coruña 7.32 & & & \\
\hline \multirow[t]{3}{*}{ E. indagador } & U.Vigo 6.67 & \multirow[t]{3}{*}{.011} & \multirow[t]{3}{*}{.989} & \\
\hline & U.S.C. 6.66 & & & \\
\hline & U.Coruña 6.65 & & & \\
\hline
\end{tabular}

Tabla 4. Resultados de las pruebas de Anova y TuKey en función de la variable universidad. *La diferencia de medias es significativa al nivel de .05 .

Teniendo en cuenta la variable especialidad, comprobamos en la tabla 5 que efectivamente existen diferencias estadísticamente significativas entre algunas de las especialidades en función del estilo de enseñanza.

En líneas generales, son los estudiantes que cursan la especialidad de Educación Infantil los que manifiestas preferencias más proactivas en relación el alumnado de las otras especialidades. Así mismo, son los que menos evidencian representaciones coherentes con el estilo académico. Por el contrario, son los matriculados en la especialidad de Educación Física los que manifiestan en mayor número características 
consistentes con el estilo de enseñanza académico, siendo menos proclives al uso de estilos de enseñanza activos.

\begin{tabular}{|c|c|c|c|c|}
\hline FACTORES & Media & $\mathbf{F}$ & Sig. & HSD de TUKEY Sig.* \\
\hline \multirow{5}{*}{$\begin{array}{l}\text { Estilo } \\
\text { reflexivo }\end{array}$} & Ed. Infantil: 28.30 & \multirow[t]{5}{*}{5.081} & \multirow[t]{5}{*}{.001} & Ed.Infantil-Ed.Primaria:.002 \\
\hline & Ed. Primaria: 27.20 & & & Ed.Infantil-Ed.Física:.005 \\
\hline & Ed. Física: 26.82 & & & \\
\hline & Ed. Musical: 27.95 & & & \\
\hline & L.Extranjeras: 27.88 & & & \\
\hline \multirow{5}{*}{$\begin{array}{l}\text { Estilo } \\
\text { cooperador }\end{array}$} & Ed. Infantil: 25.65 & \multirow[t]{5}{*}{9.019} & \multirow[t]{5}{*}{.000} & Ed.Infantil-Ed.Primaria:.000 \\
\hline & Ed. Primaria:24.55 & & & Ed.Infantil-Ed.Física:.000 \\
\hline & Ed. Física:23.92 & & & Ed.Física-L.Extranjera:.011 \\
\hline & Ed. Musical:25.20 & & & \\
\hline & L.Extranjeras:25.33 & & & \\
\hline \multirow{5}{*}{$\begin{array}{l}\text { Estilo } \\
\text { académico }\end{array}$} & Ed. Infantil:11.93 & \multirow[t]{5}{*}{10.30} & \multirow[t]{5}{*}{.000} & Ed.Infantil-Ed.Primaria:.000 \\
\hline & Ed. Primaria: 13.58 & & & Ed.Infantil-Ed.Física:.000 \\
\hline & Ed. Física:14.38 & & & Ed.Física-Ed.musical:.014 \\
\hline & Ed. Musical:12.30 & & & Ed.Física-L.Extranjeras:.026 \\
\hline & L.Extranjeras:12.62 & & & \\
\hline \multirow{5}{*}{$\begin{array}{l}\text { Estilo } \\
\text { individualizado }\end{array}$} & Ed. Infantil:17.37 & \multirow[t]{5}{*}{8.366} & \multirow[t]{5}{*}{.000} & Ed.Infantil-Ed.Primaria:.000 \\
\hline & Ed. Primaria: 16.47 & & & Ed.Infantil-Ed.Física:.000 \\
\hline & Ed. Física:15.98 & & & Ed.Infantil-Ed.Musical:.031 \\
\hline & Ed. Musical:16.36 & & & \\
\hline & L.Extranjeras: 16.80 & & & \\
\hline \multirow{5}{*}{$\begin{array}{l}\text { Estilo } \\
\text { innovador }\end{array}$} & Ed. Infantil:7.54 & \multirow[t]{5}{*}{7.190} & \multirow[t]{5}{*}{.000} & Ed.Infantil-Ed.Primaria:.032 \\
\hline & Ed. Primaria:7.30 & & & Ed.Física-Ed.Infantil:.000 \\
\hline & Ed. Física:6.95 & & & Ed.Física-Ed.Primaria:.032 \\
\hline & Ed. Musical:7.41 & & & Ed.Física-Ed.Musical:.040 \\
\hline & L. Extranjeras:7.49 & & & Ed.Física-L.Extranjera:.003 \\
\hline \multirow{5}{*}{$\begin{array}{l}\text { Estilo } \\
\text { indagador }\end{array}$} & Ed. Infantil:6.92 & \multirow[t]{5}{*}{9.768} & \multirow[t]{5}{*}{.000} & Ed.Infantil-Ed.Primaria:.000 \\
\hline & Ed. Primaria: 6.53 & & & Ed.Infantil-Ed.Física:.000 \\
\hline & Ed. Física:6.20 & & & Ed.Infantil-Ed.Musical:.012 \\
\hline & Ed. Musical:6.41 & & & Ed.Física-L.Extranjeras:.014 \\
\hline & L. Extranjeras: 6.73 & & & \\
\hline
\end{tabular}

Tabla 5. Resultados de las pruebas de Anova y HSD de Tukey en función de la variable especialidad.

*La diferencia de medias es significativa al nivel de .05 . 
El análisis en función del sexo pone de manifiesto la preferencia de estrategias basadas en metodologías de naturaleza pragmática y activa por las estudiantes. En cuanto a los estilos académico, los hombres son los recogen en su tendencia un mayor rasgo de comportamientos acordes con prácticas tradicionales.

\begin{tabular}{|c|c|c|c|}
\hline FACTORES & Media & $\mathbf{T}$ & Sig. \\
\hline \multirow[t]{2}{*}{ Estilo reflexivo } & Hombre: 26.65 & -4.191 & .000 \\
\hline & Mujer : 27.99 & & \\
\hline \multirow[t]{2}{*}{ Estilo cooperador } & Hombre: 23.99 & -5.059 & .000 \\
\hline & Mujer: 25.32 & & \\
\hline \multirow[t]{2}{*}{ Estilo académico } & Hombre: 14.09 & 4.414 & .000 \\
\hline & Mujer: 12.49 & & \\
\hline \multirow[t]{2}{*}{ Estilo individualizador } & Hombre: 15.91 & -4.962 & .000 \\
\hline & Mujer: 17.03 & & \\
\hline \multirow[t]{2}{*}{ Estilo innovador } & Hombre: 7.07 & -4.496 & .000 \\
\hline & Mujer : 7.47 & & \\
\hline \multirow[t]{2}{*}{ Estilo indagador } & Hombre : 6.29 & -4.517 & .000 \\
\hline & Mujer : 6.75 & & \\
\hline
\end{tabular}

Tabla 6. Estadísticos en función del sexo

\begin{tabular}{|c|c|c|c|c|}
\hline FACTORES & Media & $\mathbf{F}$ & Sig. & HSD de TUKEY Sig. \\
\hline \multirow{3}{*}{$\begin{array}{l}\text { Estilo } \\
\text { reflexivo }\end{array}$} & 18-20 años: 27.61 & \multirow[t]{3}{*}{3.324} & \multirow[t]{3}{*}{.037} & \multirow{3}{*}{$\begin{array}{l}* \text { Diferencias entre } 21-23 \text { y } 24 \text { o más } \\
=\operatorname{sig} .038\end{array}$} \\
\hline & 21-23 años: 27.57 & & & \\
\hline & 24 o más: 28.42 & & & \\
\hline \multirow{3}{*}{$\begin{array}{l}\text { Estilo } \\
\text { cooperador }\end{array}$} & 18-20 años: 25.12 & \multirow[t]{3}{*}{4.94} & \multirow[t]{3}{*}{.007} & \multirow{3}{*}{$\begin{array}{l}\text { *Diferencias entre } 21-23 \text { y } 240 \text { más } \\
=\operatorname{sig} .05\end{array}$} \\
\hline & 21-23 años: 24.79 & & & \\
\hline & 24 o más: 25.68 & & & \\
\hline \multirow{3}{*}{$\begin{array}{l}\text { Estilo } \\
\text { académico }\end{array}$} & 18-20 años: 12.88 & \multirow[t]{3}{*}{1.145} & \multirow[t]{3}{*}{.319} & \\
\hline & 21-23 años: 12.89 & & & \\
\hline & 24 o más: 12.33 & & & \\
\hline \multirow{3}{*}{$\begin{array}{l}\text { Estilo } \\
\text { individualizado }\end{array}$} & 18-20 años: 16.86 & \multirow[t]{3}{*}{.563} & \multirow[t]{3}{*}{.570} & \\
\hline & 21-23 años: 16.73 & & & \\
\hline & 24 o más: 16.98 & & & \\
\hline \multirow{3}{*}{$\begin{array}{l}\text { Estilo } \\
\text { innovador }\end{array}$} & 18-20 años: 7.40 & \multirow[t]{3}{*}{1.513} & \multirow[t]{3}{*}{.221} & \\
\hline & 21-23 años: 7.34 & & & \\
\hline & 24 o más: 7.51 & & & \\
\hline \multirow{3}{*}{$\begin{array}{l}\text { Estilo } \\
\text { indagador }\end{array}$} & $18-20$ años: 6.75 & \multirow[t]{3}{*}{1.040} & \multirow[t]{3}{*}{.354} & \\
\hline & 21-23 años: 6.62 & & & \\
\hline & 24 o más: 6.63 & & & \\
\hline
\end{tabular}

Tabla 7. Resultados en las pruebas de Anova y Tukey en función de la variable edad. *La diferencia de medias es significativa al nivel de 0.05 . 
Al plantearnos qué estilos de enseñanza se manifiestan en las preferencias de los alumnos de Ciencias de la Educación en relación con la edad que poseen, cobra especial relevancia los comportamientos de carácter activo destinados a favorecer la reflexión, el análisis de la información, la producción de actitudes que facilitan el aprendizaje autónomo y la interacción entre los alumnos, en los estudiantes de magisterio con edades superiores a los 24 años.

\section{Discusión y conclusiones}

En la búsqueda constante de un aprendizaje efectivo las investigaciones se han orientado más hacia el estudio de las estrategias de aprendizaje que en el de los estilos de enseñanza (Gargallo López, Suárez Rodríguez y Ferreras Remesal, 2007; Marín Gracia, 2002; Ruiz Lara, Hernández Pina y Ureña Villanueva, 2008). No obstante, anteriormente recogíamos la existencia de algunos estudios en los que se focalizaba el interés en tipologías dicotómicas, generalmente contraponiendo un estilo activo y otro académico y en su mayoría llevados a cabo en otros contextos educativos. En este sentido, Hood, Poulson, Mason, Walker y Dixon (2009) a diferencia de los otros autores, sostienen que tanto el estilo transformacional (activo) como el transaccional (académico-tradicional) tienen lugar en el aula y que ambos pueden ayudar a crear un ambiente de aprendizaje positivo.

Delgado (1992) elabora una taxonomía que parte de las relaciones acaecidas dentro del aula, de la distribución de poder y de la concepción del currículo entre otros aspectos. Se trata de una propuesta en la que se respetan los estilos de enseñanza difundidos por Mosston y Ashworth (1990) con la introducción de algunas modificaciones. El autor clasifica los estilos de enseñanza en: tradicional, individualizador, participativo, socializador, cognoscitivo y creativo. Los resultados de su investigación indican una clara tendencia hacia estilos activos y una valoración negativa hacia estilos tradicionales. En una investigación posterior, Delgado (1998) estudia los estilos de enseñanza en estudiantes en formación inicial de Educación Física y profesores en ejercicio de su profesión. En ambos casos hay un claro predominio de los estilos activos frente a los estilos tradicionales.

Sin embargo, Som Castillo y Muros Molina (2007) tras los resultados obtenidos en un cuestionario aplicado a profesores en activo, destacan que el estilo de enseñanza tradicional es el más conocido y el que mayor satisfacción le ha dado a los profesores tras su aplicación, seguido del estilo de enseñanza participativo y del individualizador. Es el estilo de enseñanza cognoscitivo el que peor satisfacción les ha proporcionado, seguido del estilo de enseñanza creativo y socializador.

Los resultados del presente estudio indican que, cuando se analiza la totalidad de la muestra, existe una clara predominancia de los estilos activos. Básicamente los estudiantes reflejan mayor preferencia por el estilo innovador, cooperativo y reflexivo. Consecuentemente parece perfilarse una creencia acorde con estilos potenciadores de la espontaneidad, la capacidad productiva, el trabajo en grupo y de situaciones que fomenten la solución de problemas, el análisis y la toma de decisiones. Autores como 
Coldren y Hively, 2009; Hood, 2009 y Pierro, 2009 sustentan que un estilo activo genera más estimulación en los discentes.

A su vez, las derivaciones de este trabajo apuntan a que la Universidad modula la aparición de estilos de enseñanza preferentes entre sus alumnos. Se han observado diferencias significativas entre la Universidad de Vigo y la Universidad de Santiago en los estilos reflexivos y académicos. La USC presenta una mayor tendencia hacia el estilo reflexivo y una menor preferencia hacia estilos académicos tradicionales. Esto implica que la formación inicial que el alumnado de magisterio recibe en la Universidad, puede influir en su concepción sobre el estilo de enseñanza. De ahí la importancia de que durante la Formación Inicial el Profesorado Universitario se favorezca la adopción de estrategias y técnicas activas mediante su utilización en el aula.

Cuando se realiza un análisis multivariante teniendo en cuenta las especialidades, se comprueba que esta variable resulta ser la que mayores diferencias presenta con respecto a otras categorías, siendo la especialidad de Educación Física la que registra una media superior de tendencias hacia el estilo academicista. Resultado que no coincide con el expresado por Delgado (1998) quién apunta que la tendencia de los estudiantes de la especialidad de Educación Física es clara hacia la valoración negativa de los estilos de enseñanza tradicionales. Disposición que puede verse propiciada por tratarse de un área que fomenta un aprendizaje eficaz de habilidades dentro de unos parámetros de rigor, orden y competitividad, marcado esencialmente por las pautas del profesor que plantea un modelo de ejecución a imitar. Contrariamente vemos como las representaciones asociadas a estilos activos han sido las que en mayor medida han identificado a los alumnos de la especialidad de Educación Infantil, manifestando las puntuaciones más bajas en relación al estilo académico.

Así mismo, la variable sexo, parece tener un gran peso respecto a las diferencias de estilos, siendo las mujeres las que muestran una mayor predilección por estilos de enseñanza más coherentes con postulados activos, mientras que el estilo académico predomina en los hombres. Por ello, creemos fundamental introducir estudios de las relaciones entre estas dimensiones y las variables psicológicas, incluyendo factores sociodemográficos que mediarían en esta relación. La comparación entre varones y mujeres sin tener en cuenta otras variables puede llevar a extraer conclusiones sesgadas.

Respecto a la edad, los futuros profesores cuanto mayor son, más concienciados parecen estar en el uso de una enseñanza activa.

En consecuencia, se infiere que la universidad, especialidad, sexo y edad influyen, en la configuración de un estilo de enseñanza peculiar, además de factores exógenos y endógenos al estudiante como la personalidad, vida académica y contexto, entre otros mencionados con anterioridad

Estas conclusiones implican que durante la formación inicial, es preciso favorecer programas de intervención educativa que conlleven la adopción, por parte del futuro profesor, de un estilo propio de carácter activo. Formación del profesorado y estilos de enseñanza son dos aspectos importantes en el desarrollo docente. Formación, entendida 
como una actividad que conduce a la excelencia profesional en la medida en que se pretende que docentes en ejercicio y en período de capacitación adquieran conocimientos, destrezas y roles adecuados para el desempeño de una enseñanza de calidad. En este marco, es labor de los formadores dotarles de las herramientas conceptuales necesarias para problematizar, reflexionar, buscar nuevos significados sobre enfoques, métodos, actitudes, con el fin de adoptar la decisión más correcta en función del contexto y las características individuales del alumnado, propiciando el desarrollo de sujetos innovadores, reflexivos, críticos y creativos. Los escenarios educativos deben consolidar y estimular el uso de diferentes estilos como ejercicio de integración y no de segmentación de la praxis docente.

Como nota final, en líneas generales en la muestra estudiada, se cumple el principio clave de integración de estilos y flexibilidad. Aspecto que refuerza significativamente el uso de estilos de enseñanza mixtos, aunque uno de ellos sea el preferente, ofreciendo oportunidades a todos los alumnos, independientemente de cuál sea su estilo de aprendizaje, respetando las diferencias individuales. Se hace imprescindible que, el futuro docente sea formado en metodologías activas y conducido en la búsqueda de estilos de enseñanza que le dote de los mecanismos necesarios para ser un verdadero agente de cambio, y a su vez, lo faculte para interactuar dentro de un marco de colaboración y actuación que le posibilite la toma de decisiones concretas que conecten con un espacio multicultural y cambiante.

Consideramos que los resultados obtenidos abren nuevas vías para seguir avanzando en los estilos de enseñanza preferentes en entornos docentes haciendo hincapié en los indicadores que permitan verificar la eficacia de los estilos de enseñanza implementados atendiendo a las variables consideradas en el presente trabajo. Pero también, dirigiendo la investigación hacia el análisis de la posible vinculación entre los estilos de enseñanza y variables como el estilo de aprendizaje, el rendimiento académico, el estilo motivacional o el autoconcepto.

\section{Referencias bibliográficas}

AGUILERA, E Y ORTIZ, E. (2010). La caracterización de perfiles de estilos de aprendizaje en la Educación Superior, una visión integradora. Revista Estilos de Aprendizaje, 5 (5), 26-41

ALONSO, C. (2008). "Estilos de aprendizaje. Presente y futuro". Revista de Estilos de aprendizaje.1, 4-15. Recuperado de

http://www.uned.es/revistaestilosdeaprendizaje/numero_1/1sr_1_abril_2008.pdf Consultado el 7 de julio 2014.

ALONSO, C.; GAlleGO, D. J.; HONEY, P. (1999). Los estilos de aprendizaje. Procedimientos de diagnóstico y mejora. Bilbao: Ediciones Mensajero.

BALLESTA, F., IZQUIERDO, T., ROMERO, B. (2011). Percepción del alumnado de pedagogía ante el uso de las metodologías activas. Educatio Siglo XXI, 29 (2), 353368. 
CABRERA, L.; BETHENCOURT, J.; GONZÁLEZ, M.; Y ÁVAREZ, P. (2006). "Un estudio transversal retrospectivo sobre prolongación y abandono de estudios universitarios". Revista Electrónica de Investigación y Evaluación Educativa, 12 (1). Recuperado de http://www.uv.es/RELIEVE/v12n1/RELIEVEv12n1_1.htm Consultado el 3 de noviembre de 2013.

COLDREN, J. \& HIVELY, J. (2009). Interpersonal teaching style and student impression formation. College Teaching, 57 (2), 93-98.

DARLING-HAMMOND, L. (2000). Teacher Quality and Student Achievement: A Review of State Policy Evidence. Educational Policy Analysis Archives, 8(1).

DELGADO, M. A. (1992). Los Estilos de Enseñanza en la Educación Física. Propuesta para una reforma de la enseñanza. Granada: Universidad de Granada.

DELGADO, M. A. (1996). Aplicaciones a los Estilos de Enseñanza en la Educación Primaria. En C. Romero (comp.), Estrategias Metodológicas para el aprendizaje de los contenidos de Educación Física Escolar (73-86). Granada: Universidad de Granada.

DELGADO, M. A. (1998). Comparación de la valoración de los Estilos de Enseñanza por los futuros profesores de Educación Física durante la Formación Inicial y Profesores de Educación Física en Formación. Lecturas de Educación Física. Recuperado de http://w.w.w.sir.ca/revista/ Consultado el 3 de noviembre de 2013.

GALLEGO GIL, D. Y NEVOT LUNA, A. (2008). Los estilos de aprendizaje y la enseñanza de las matemáticas. Revista Complutense de Educación, 19 (1) 95-112

GARCÍA-BERRO, E., ROCA, S., AMBLAS, G., MURCIA, F., SALLARES, J., Y BUGEDA, G. (2010). La evaluación de la actividad docente del profesorado en el marco del EEES. Aula Abierta, 38, 29-40.

GARGALLO, B. (2008). Estilos de docencia y evaluación de los profesores universitarios y su influencia sobre los modos de aprender de sus estudiantes. Revista Española de Pedagogía, 241, 425-446.

GARGALLO LÓPEZ, B., SUÁREZ RODRÍGUEZ, J. Y FERRERAS REMESAL, A. (2007). Estrategias de aprendizaje y rendimiento académico en estudiantes universitarios. Revista de Investigación Educativa, 25 (2), 421-441.

GARGALLO, B.; SÁNCHEZ, F.; ROS, C. Y FERRERAS, A. (2010). Estilos docentes de los profesores universitarios. La percepción de los alumnos de los buenos profesores. Revista Iberoamericana de Educación, 51(4).

GONZÁLEZ-PEITEADO, M. (2012). Las representaciones sobre los estilos de enseñanza en la construcción del rol docente. Tesis doctoral no publicada. Universidad de Vigo.

GONZÁLEZ-PEITEADO, M. (2013). Los estilos de enseñanza y aprendizaje como soporte de la actividad docente. Revista Estilos de Aprendizaje. 11, 51-70 
GONZÁLEZ-PEITEADO, M.; LÓPEZ-CASTEDO, A. Y PINO-JUSTE, M. (2013). Análisis psicométrico de una Escala sobre Estilos de Enseñanza (ESEE). Revista Enseñanza \& Teaching, 31(1) 181-198.

GONZÁLEZ-PEITEADO, M. Y PINO-JUSTE, M (2014). Aproximación a las representaciones y creencias del alumnado de magisterio sobre los estilos de enseñanza. Revista Educación XX1, 17 (1), 81-110. doi: 10.5944/educxx1.17.1.10706.

GONZÁLEZ-PEITEADO, M. Y RODRÍGUEZ-LÓPEZ, B. (2014).La formación inicial de los profesores de Lengua Extranjera: un espacio para generar estilos de actuación. Revista Bordón 66 (4) 69-85

GRAVINI, M.; CABRERA, E.; ÁVILA, V. Y VARGAS, I. (2009). Estrategias de enseñanza en docentes y estilos de aprendizaje en estudiantes del programa de psicología de la Universidad Simón Bolívar, Barranquilla. Revista de Estilos de Aprendizaje, 3, 124-140.

HERVÁS AVILES R.M. (2005). Estilo de enseñanza y aprendizaje en escenarios educativos. Granada: Grupo Editorial Universitario.

HOOD, J., POULSON, R., MASON, S., WALKER, T. Y DIXON, J. (2009). An examination of traditional and nontraditional students' evaluations of professorial leardership styles: transformational versus transactional approach. Journal of the Scholarship of Teaching and Learning, 9 (1), 1-12.

LÓPEZ-AGUADO, M. (2011). Estilos de aprendizaje. Diferencias por género, curso y titulación. Revista de estilos de aprendizaje, 8 (7) 109-134.

MARTÍNEZ GEIJO. P. (2007) Aprender y enseñar: Los estilos de aprendizaje y de enseñanza desde la práctica del aula. Bilbao: España.Martínez Geijo, P. (2008). Estilos de aprendizaje: pautas metodológicas para trabajar en el aula. Revista Complutense de Educación 19 (1) 77-94

MICHEL, N., JAMES, J. \& VARELA, O. (2009). Active versus passive teaching styles: an empirical study of student learning outcomes. Human Resource Development Quarterly, 20 (4), 397-418.

MOSSTON, M. Y ASHWORTH, S. (1990-1993). La enseñanza de la Educación Física. La reforma de los Estilos de Enseñanza. Barcelona: Hispano Europea.

MARÍN GRACIA, M. A. (2002). La investigación sobre diagnóstico de los estilos de aprendizaje en la enseñanza superior. Revista de Investigación Educativa, 20 (2), 303-337.

PIERRO, A., PRESAGHI, F., HIGGINS, T. \& KRUGLANSKI, A. (2009). Regulatory mode preferentes for autonomy supporting versus controlling instructional styles. British Journal of Educational Psychology, 79, 599-615.

RAPOSO, R.; BARCIA, E.; FERNÁNDEZ-CARBALLIDO, A.; MONTEJO, C.; NEGRO, S. Y GALLEGO, D. (2004). Estilos de aprendizaje en las facultades de 
Farmacia. Actas del I Congreso Internacional de Estilos de Aprendizaje. Madrid: UNED.

RUIZ LARA, E., HERNÁNDEZ PINA, F. Y UREÑA VILLANUEVA, F. (2008). Enfoques de aprendizaje y rendimiento institucional y afectivo de los alumnos de la titulación de ciencias de la actividad física y del deporte. Revista de Investigación Educativa, 26 (2), 307-322.

SOM CASTILLO, J.; MUROS MOLINA, J.; PASCUAL ALVAREZ, J.; LEYVA RODRIGUEZ, A.; MEDINA CASAUBÓN, J. (2007). Conocimiento que tiene el profesorado del área de Educación Física de secundaria obligatoria y bachillerato de Granada sobre los estilos de enseñanza. Recuperado de http://www.efdeportes.com/ Revista Digital - Buenos Aires - Año 12 - $\mathrm{N}^{\circ} 115$ Consultado el 3 de noviembre de 2013.

TEJADA FERNÁNDEZ, J. (2011). ¿Qué son la didáctica y la teoría del currículum? En I. Cantón Mayo y M. Pino Juste (comp.). Diseño y desarrollo del currículum (pp.19-40). Madrid: Alianza.

TRUJILlO TORRES, J.; HINOJO LUCENA, F. Y AZNAR DÍAZ, I. (2011). Propuestas de trabajo innovadoras y colaborativas e-learning 2.0 como demanda de la sociedad del conocimiento. Revista Estudios sobre Educación, 20, 141-159

WELTMAN, D. \& WHITESIDE, M. (2010). Comparing the Effectiveness of Traditional and Active Learning Methods in Business Statistics: Convergence to the Mean. Journal of Statistics Education 18, 1. Recuperado de: http://www.amstat.org/publications/jse/v18n1/weltman.pdf. (Consultado el 7 de noviembre de 2014)

\section{Correspondencia con los autores}

Margarita GONZÁLEZ-PEITEADO

Universidad Nacional de Educación a Distancia (Centro Asociado de Pontevedra)

Rúa de Portugal, 1.

36004. Pontevedra. España

e-mail: margonzalez@pontevedra.uned.es

Margarita PINO-JUSTE

Universidad de Vigo

Facultad de Ciencias de la Educación y del Deporte

Campus de A Xunqueira

36005 Pontevedra

e-mail: mpino@uvigo.es 